\title{
Erratum to: The open access advantage considering citation, article usage and social media attention
}

\author{
Xianwen Wang ${ }^{1}$ Chen Liu ${ }^{1} \cdot$ Wenli Mao ${ }^{1}$ Zhichao Fang ${ }^{1}$
}

\section{Erratum to: Scientometrics DOI 10.1007/s11192-015-1547-0}

Because the article views data of a few Nature Communications papers have unusual dramatic step changes, for example http://www.nature.com/ncomms/journal/v3/n1/ ncomms1655/metrics, in the paper 10.1007/s11192-015-1547-0, 18 papers (about $1 \%$ of the total samples) with massive jumps are excluded and not plotted in Fig. 2, which we forgot to mention in the "Data and Method" section.

The online version of the original article can be found under doi:10.1007/s11192-015-1547-0.

Xianwen Wang

xianwenwang@dlut.edu.cn; xwang.dlut@gmail.com;

http://xianwenwang.com

1 WISE Lab, Faculty of Humanities and Social Sciences, Dalian University of Technology, Dalian 116085, China 